\title{
POROELASTIC MODELING IN STRATIFIED MEDIA ACROSS ALL FREQUENCIES
}

\author{
Igor Barbosa de Oliveira ${ }^{1}$, Marcia Miranda Azeredo ${ }^{1}$, \\ Mariane Ribeiro Silva Tiradentes Miranda ${ }^{1}$ and Viatcheslav Ivanovich Priimenko ${ }^{1,2}$
}

ABSTRACT. There is considered a layered heterogeneous poroelastic isotropic medium with physical parameters characterized by piecewise constant functions of the depth only. We derive a mathematical algorithm for calculating reflected/transmitted poroelastic waves across all temporal frequencies. To define the frequency effect we use the dynamic permeability expression proposed by Johnson, Koplik and Dashen; in the time domain, this coefficient introduces order $1 / 2$ shifted fractional time derivative involving a convolution product. The algorithm proposed is based on the formalism introduced by Ursin. The algorithm is tested numerically in a 1D-case. The numerical experiments confirm the effectiveness of the proposed algorithm in identifying the main wave events in both low frequency and high frequency regimes in the reservoir and laboratory scales.

Keywords: stratified porous medium, Biot and Biot-JKD models, Ursin's formalism.

RESUMO. Neste trabalho, consideramos um meio poroelástico estratificado, isotrópico e heterogêneo com parâmetros físicos caracterizados por funções constantes por partes em relação à profundidade. Nós derivamos um algoritmo matemático para calcular as ondas poroelásticas refletidas/transmitidas em todas as frequências temporais. Para definir o efeito da frequência, usamos a expressão de permeabilidade dinâmica proposta por Johnson, Koplik e Dashen; no domínio do tempo, este coeficiente introduz a derivada do tempo fracionária de $1 / 2$ de ordem de deslocamento envolvendo um produto de convolução. 0 algoritmo proposto é baseado no formalismo introduzido por Ursin e foi testado numericamente para o caso 1D. Os experimentos numéricos confirmaram a efetividade do algoritmo na identificação dos principais eventos de onda nos regimes de baixa frequência e alta frequência, nas escalas de reservatório e laboratorial, respectivamente.

Palavras-chave: meio poroso estratificado, modelos de Biot e Biot-JKD, formalismo de Ursin.

\footnotetext{
${ }^{1}$ Universidade Estadual do Norte Fluminense Darcy Ribeiro / Laboratório de Engenharia e Exploração de Petróleo - UENF/LENEP. Rodovia Amaral Peixoto, Km 163, Imboassica - Macaé, RJ, Brazil - E-mails: igorbarbosa.lenep@gmail.com, marcia578@gmail.com, slava@lenep.uent.br, mariane_rds@hotmail.com ${ }^{2}$ National Institute of Science and Technology - Petroleum Geophysics, Salvador, BA, Brazil
} 


\section{INTRODUCTION}

Reflection and transmission of elastic waves propagating through successive layers of fluid-filled porous media is inherent to many theoretical and practical applications in geophysics and petroleum engineering, where porous media filled with fluid and/or gas is of great interest.

There are many works devoted to the development and application of analytical/semi-analytical methods for wave propagation analysis in stratified elastic media, see, for instance, Akkuratov \& Dmitriev (1984), Brekhovskih (1960), Fatianov \& Mikhailenko (1988), Fatianov (1990), Haskell (1953), Karchevsky (2005a,b), Kunetz \& D'Erceville (1962), Molotkov (1984, 2002), Ursin (1983), Thomson (1950). The development of similar methods in the case of stratified porous media (low frequency range, static permeability case) is very important too, see Allard et al. (1989), Baird et al. (1996), Molotkov (2002) and Carcione (2007).

Now, let us comment on some results in the literature concerning the poroelastic modeling in stratified media across all frequencies (dynamic permeability case). Carcione (1996) presented a finite-differencing approach that allows for the dynamic permeability by approximating it as a sum of Zener relaxation functions. Hanyga \& Lu (2005) designed a numerical method based on the combination of the Fourier pseudo-spectral and predictor-corrector methods. Masson \& Pride (2010) used an explicit time-stepping finite-difference scheme for solving Biot's equations of poroelasticity across the entire band of frequencies. Blanc (2013) proposed an explicit finite-difference scheme based on the diffusive representation of fractional derivatives when the convolution kernel is replaced by a finite number of memory variables that satisfy local-in-time ordinary differential equations. Li et al. (2015) proposed a new algorithm based on the rational expansion of dynamic permeability and the combination of the generalized phase-shift scheme and the pseudo-spectral method. Recently, Milani et al. (2016) presented a finite-element technique to solve the one-dimensional Biot equations (with the dynamic permeability) in the space-frequency domain; there was considered the case of a medium composed of periodically distributed mesoscopic layers. A review of the various techniques and discussion of the numerical implementation aspects for application to seismic modeling and rock physics, as for instance the role of Biot's diffusion wave as a loss mechanism and interface waves in porous media, was done in Carcione et al. (2010).

We now comment on some theoretical results concerning existence, uniqueness and continuous dependence of the solution to Eqs. (1)-(3). To our knowledge the only paper about the subject was the paper Lorenzi \& Priimenko (2014), where the authors studied well-posedness of an initial boundary-value problem. As a result of the investigation, the authors proved a uniqueness and continuous dependence result for a generalization of Eqs. (1)-(3) related to a general bounded open set $\Omega$ in any spatial dimension $n=1,2,3$, both on the unbounded time interval $(0,+\infty)$ and on the bounded time interval $(0, T)$; however, the existence question remains open.

In this paper, we derive the mathematical basis for an efficient computer code for poroelastic modeling in stratified media across all frequencies. Our method is based on a formalism introduced by Ursin (1983), who gives a unified treatment of electromagnetic waves, acoustic waves, and the isotropic elastic waves in plane layered media. We apply Ursin's formalism for the case of a stack of homogeneous layers, that is, when the material parameters are piecewise constant functions of depth. In this case many quantities can be computed with explicit algebraic formulas which can then be made the basis of a fast computer code.

In 2006 this formalism was applied to the Pride equations for simulation of the electrokinetic phenomena in layered media, see White \& Zhou (2006). Although the results obtained by White and Zhou allow, under certain conditions, to split Pride's equations and select only the poroelastic part, we examine the case of a more complete poroelastic system, characterized by presence in the Darcy law of an inertial force connected with the effective density of pore fluid and by dynamic permeability introduced by Johnson, Koplik and Dashen, see Miranda (2016) and Oliveira (2018) for details. For the low-frequency range a similar algorithm was formulated by Azeredo (2013) and Azeredo \& Priimenko (2015).

This paper is organized as follows. The original Biot-JKD model is briefly outlined and then we state our problem. We give a self-contained derivation of Ursin's diagonalization method, derive formulas for propagator matrices, jump matrices, and reflection/transmission matrices, coupling the results with general sources and boundary conditions. Some numerical experiments performed with realistic values of the physical parameters are described. Finally, the discussion of the results obtained and the conclusions and some futures lines of research are presented.

\section{GOVERNING EQUATIONS}

In 1956, Biot presented a theory of poroelasticity, which forms the basis of most investigations into elastic wave propagation 
in porous media containing a fluid. However, first works on poroelastodynamics are those of Frenkel (1944). Further work in the Russian scientific community based on this pioneering work is reviewed in Nikolaevskiy (2005). The connection of Frenkel's work to the Biot theory is presented in Pride \& Carambois (2005), where it is shown that both researchers have developed the same theory. The theory of Biot predicts two bulk compressional waves and one shear wave, which are dispersive and dissipative. The second bulk compressional wave, also known as the slow wave of Biot, was experimentally observed in a water-saturated porous solid by Plona (1980) using an ultrasonic mode conversion technique.

For the higher frequency range, Biot (1956) presented a formula for the dynamic permeability with two particular types of pore geometry: two-dimensional flow between parallel walls and three-dimensional flow in a circular duct. Modeling this dynamic permeability behavior along with finding proper microstructural pore-space descriptors has received considerable attention in the literature, see Auriault et al. (1985). Perhaps the most popular dynamic permeability model has been suggested in Johnson et al. (1987). They published a general expression for the dynamic permeability in the case of random pores with constant radii, leading to the so-called Biot-JKD model. In this model, viscous stresses depend on the square root of the temporal frequency; the only additional parameter is the viscous characteristic length $\Lambda$.

Using the dynamic permeability proposed by Johnson, Koplik and Dashen, we can rewrite the Biot system in the Biot-JKD form

$$
\begin{aligned}
& \rho \partial_{t}^{2} u+\rho_{f} \partial_{t}^{2} w=\nabla \cdot \tau+f, \\
& \rho_{f} \partial_{t}^{2} u+\rho_{w} \partial_{t}^{2} w+\frac{\eta}{\kappa_{0}} h *\left[\partial_{t}^{2} w+\omega_{c} \partial_{t} w\right]=-\nabla p+g,
\end{aligned}
$$

with the following constitutive laws (isotropic media case):

$$
\begin{aligned}
& \tau=(\lambda \nabla \cdot u+c \nabla \cdot w) I+\mu\left(\nabla u+\nabla u^{T}\right), \\
& p=-c \nabla \cdot u-m \nabla \cdot w,
\end{aligned}
$$

where the following notations were introduced:

$$
\begin{aligned}
& \rho_{w}=F_{e} \rho_{f}, \rho=\phi \rho_{f}+(1-\phi) \rho_{s}, P=\frac{4 a \kappa_{0}}{\phi \Lambda^{2}}, \omega_{c}=\frac{2 \pi f_{c}}{P}, \\
& h(t)=\frac{e^{-\omega t}}{\sqrt{\pi \omega_{c} t}}, h * z(t, \cdot)=\int_{0}^{t} h(t-s) z(s, \cdot) d s .
\end{aligned}
$$

This model involves the following functions and physical parameters: the elastic stress tensor $\tau$ and the acoustic pressure $p$, the relative displacement vector $w=\left(w_{1}, w_{2}, w_{3}\right)^{T}$ of the fluid phase, the volume density of the body force for the pore fluid $g=\left(g_{1}, g_{2}, g_{3}\right)^{T}$, the density $\rho_{f}$ and the dynamic viscosity $\eta$ of the fluid; the displacement vector $u=\left(u_{1}, u_{2}, u_{3}\right)^{T}$ of the solid phase, the volume density of the body force for the saturated porous medium $f=\left(f_{1}, f_{2}, f_{3}\right)^{T}$; the porosity $0<\phi<1$, the electrical formation factor $F_{e}$, the steady-flow limit of the permeability $\kappa_{0}$, the density $\rho_{s}$ and the Lamé coefficients $\lambda, \mu$ of the elastic skeleton, and the two Biot coefficients $c$ and $m$ of the saturated matrix; $\omega_{c}$ is the circular frequency at which viscous boundary layers first develop, $f_{c}$ is the transition frequency, and $P$ is the Pride number; $I$ is the $3 \times 3$-identity matrix. In (1) the convolution term denotes the viscous dissipation induced by the relative motion between the fluid and the elastic skeleton.

\section{STATEMENT OF THE PROBLEM}

We shall consider wave propagation in a porous medium $\mathscr{R}=$ $\cup_{k=0}^{N} \mathscr{R}_{k}$, composed by stratified layers identified with $\mathscr{R}_{k}=$ $\left\{x=\left(x_{1}, x_{2}, x_{3} \equiv z\right) \in \mathbb{R}^{3}: z_{k}<z<z_{k+1}\right\}$, with $0=$ $z_{0}<z_{1} \cdots<z_{N+1}=\infty$. The Biot-JKD equations (1)-(3) in the temporal frequency $(\omega)$ domain, at each point $x \in \mathscr{R}$, are (time dependence of $e^{-i \omega t}$ is assumed)

$$
\begin{aligned}
& -i \omega\left(\rho v+\rho_{f} q\right)=\nabla \cdot \tau+f, \\
& -i \omega\left(\rho_{f} v+\rho_{w} q\right)+d(\omega) q=-\nabla p+g, \\
& -i \omega \tau=(\lambda \nabla \cdot v+c \nabla \cdot q) I+\mu\left(\nabla v+\nabla v^{T}\right), \\
& -i \omega p=-c \nabla \cdot v-m \nabla \cdot q,
\end{aligned}
$$

where $v=-i \omega u, q=-i \omega w$ are the solid and relative fluid velocities, and

$$
d(\omega)=\frac{\eta}{\kappa_{0}} \frac{1}{\sqrt{\omega_{c}}}\left(\omega_{c}-i \omega\right)^{1 / 2} .
$$

Remark 1. In the low frequency range $\lim _{\omega \rightarrow 0} d(\omega)=\eta / \kappa_{0}$, i.e., we obtain the classical low frequency variant of the Biot system.

We assume that all material parameters are represented by piece-wise constant functions depended only the depth coordinate $z$, with the discontinuities at the points $z=z_{k}, k=$ $1,2, \ldots, N$. At the internal layer boundaries $z=z_{k}$, we suppose that the following functions are continuous:

$$
v, q, p, \tau_{13}, \tau_{23}, \tau_{33} .
$$

The boundary conditions at the free surface $z=0$ are

$$
p=\tau_{13}=\tau_{23}=\tau_{33}=0 .
$$


And finally, at the infinity the solution satisfy the following radiation conditions:

$$
\lim _{|x| \rightarrow \infty}(v, q)=0 .
$$

\section{METHOD}

\section{Special format}

Consider the Fourier transform in the two coordinates $x_{1}, x_{2}$

$$
\hat{X}\left(k_{1}, k_{2}, z\right)=F_{x_{1} x_{2}}(X) \equiv \int_{\mathbb{R}^{2}} e^{-i\left(k_{1} x_{1}+k_{2} x_{2}\right)} X\left(x_{1}, x_{2}, z\right) d x_{1} d x_{2} .
$$

Let $\left(k_{1}, k_{2}\right)^{T}$ be the horizontal wave number and $k=\sqrt{k_{1}^{2}+k_{2}^{2}}, \gamma=k \omega^{-1}$. Applying the Fourier transform to Eqs. (4) we obtain the system of ordinary differential equations (ODE's) represented in the terms of $\hat{f}, \hat{g}, \hat{v}, \hat{q}, \hat{\tau}, \hat{p}$. The ODE's obtained after application of the Fourier transform can be simplified if we define

$$
\tilde{v}=\Omega \hat{v}, \tilde{q}=\Omega \hat{q}, \tilde{\tau}=\Omega \hat{\tau} \Omega^{T}, \tilde{p}=\hat{p}, \tilde{f}=\Omega \hat{f}, \tilde{g}=\Omega \hat{g},
$$

where

$$
\Omega=\frac{1}{k}\left(\begin{array}{ccc}
k_{1} & k_{2} & 0 \\
-k_{2} & k_{1} & 0 \\
0 & 0 & k
\end{array}\right) .
$$

A straightforward calculation uncouples this system

$$
\frac{d \Phi^{(m)}}{d z}=-i \omega M^{(m)} \Phi^{(m)}+S^{(m)}, m=1,2
$$

where $\Phi^{(m)}, m=1,2$, are the $2 n_{m}$-vectors $\left(n_{1}=3, n_{2}=1\right)$ defined as

$$
\Phi^{(1)}=\left(\tilde{v}_{3}, \tilde{\tau}_{13},-\tilde{q}_{3}, \tilde{\tau}_{33}, \tilde{v}_{1}, \tilde{p}\right)^{T}, \Phi^{(2)}=\left(\tilde{v}_{2}, \tilde{\tau}_{23}\right)^{T},
$$

$S^{(m)}$ are the source $2 n_{m}$-vectors, and $M^{(m)}$ are the $2 n_{m} \times 2 n_{m}$-matrices

$$
M^{(m)}=\left(\begin{array}{cc}
0 & M_{1}^{(m)} \\
M_{2}^{(m)} & 0
\end{array}\right)
$$

with symmetric $n_{m} \times n_{m}$-matrices $M_{1}^{(m)}, M_{2}^{(m)}$. For Systems 1 and 2 the sub-matrices and the corresponding source vectors are

$$
\begin{gathered}
M_{1}^{(1)}=\left(\begin{array}{ccc}
-\beta m & \beta \gamma\left(c^{2}-\lambda m\right) & -\beta c \\
\beta \gamma\left(c^{2}-\lambda m\right) & \rho+\frac{i \omega \rho_{f}^{2}}{d-i \omega \rho_{\omega}}-4 \beta \gamma^{2} \mu\left(c^{2}-m(\lambda+\mu)\right) & 2 \beta \gamma \mu c-\frac{i \omega \rho_{f} \gamma}{d-i \omega \rho_{\omega}} \\
-\beta c & 2 \beta \gamma \mu c-\frac{i \omega \rho_{f} \gamma}{d-i \omega \rho_{\omega}} & -\beta(\lambda+2 \mu)+\frac{i \omega \gamma^{2}}{d-i \omega \rho_{\omega}}
\end{array}\right) \\
M_{2}^{(1)}=\left(\begin{array}{ccc}
\rho & \gamma & -\rho_{f} \\
\gamma & \mu^{-1} & 0 \\
-\rho_{f} & 0 & -\frac{d-i \omega \rho_{\omega}}{i \omega}
\end{array}\right), S^{(1)}=\left(0,-\tilde{f}_{1}-\frac{i \omega \rho_{f}}{d-i \omega \rho_{\omega}} \tilde{g}_{1}, \frac{i k}{d-i \omega \rho_{\omega}} \tilde{g}_{1},-\tilde{f}_{3}, 0, \tilde{g}_{3}\right)^{T}
\end{gathered}
$$


and

$$
M_{1}^{(2)}=\mu^{-1}, M_{2}^{(2)}=\rho-\mu \gamma^{2}+\frac{i \omega \rho_{f}^{2}}{d-i \omega \rho_{\omega}}, S^{(2)}=\left(0,-\tilde{f}_{2}-\frac{i \omega \rho_{f}}{d-i \omega \rho_{\omega}} \tilde{g}_{2}\right)^{T}
$$

Here $\beta=\left(c^{2}-m(\lambda+2 \mu)\right)^{-1}$. Once $\Phi^{(1)}$ and $\Phi^{(2)}$ have been determined, we may compute

$$
\begin{aligned}
& \tilde{\tau}_{11}=\beta\left(-4 \gamma \mu\left(c^{2}-m(\lambda+\mu)\right) \tilde{v}_{1}+\left(c^{2}-\lambda m\right) \tilde{\tau}_{33}+2 \mu c \tilde{p}\right), \\
& \tilde{\tau}_{22}=\beta\left(-2 \gamma \mu\left(c^{2}-\lambda m\right) \tilde{v}_{1}+\left(c^{2}-\lambda m\right) \tilde{\tau}_{33}+2 \mu c \tilde{p}\right), \\
& \tilde{q}_{1}=\frac{1}{d-i \omega \rho_{\omega}}\left(-i k \tilde{p}+i \omega \rho_{f} \tilde{v}_{1}+\tilde{g}_{1}\right), \tilde{q}_{2}=\frac{1}{d-i \omega \rho_{\omega}}\left(i \omega \rho_{f} \tilde{v}_{2}+\tilde{g}_{2}\right), \tilde{\tau}_{12}=-\mu \gamma \tilde{v}_{2} .
\end{aligned}
$$

The boundary conditions for Systems 1 and 2 at the free surface $z=0$ are

$$
\tilde{p}=\tilde{\tau}_{13}=\tilde{\tau}_{23}=\tilde{\tau}_{33}=0
$$

Note that Eqs. (12) gives $n_{1}=3$ conditions for System 1 having $2 n_{1}=6$ variables, and gives $n_{2}=1$ condition for System 2, which has $2 n_{2}=2$ variables. It means that for each system we need $n_{m}, m=1,2$ additional conditions to completely specify the solution. These relations we obtain using the radiation condition, which means that there are no up-going waves from $z=\infty$.

\section{Diagonalization}

Consider matrices of the form (8), where for simplicity we drop the superscript ${ }^{(m)}$. Assume that $M_{1} M_{2}$ has $n$ distinct nonzero eigenvalues $\lambda_{j}^{2}, j=1,2, \ldots, n$, with associated eigenvectors $a_{j}$, such that $a_{j}^{T} M_{2} a_{j}=\lambda_{j}$. Here $\lambda_{j}=\sqrt{\lambda_{j}^{2}}$ with the branch chosen so that $\operatorname{Im}\left(\lambda_{j}\right) \geq 0$ and $\lambda_{j}>0$ if $\lambda_{j}$ is real. Define $b_{j}=\lambda_{j}^{-1} M_{2} a_{j}$. This vector is an eigenvector of $M_{2} M_{1}$ with eigenvalue $\lambda_{j}^{2}$. Using symmetricity of $M_{1}, M_{2}$ we obtain $a_{j}^{T} b_{i}=\delta_{j}^{i}$, where $\delta_{j}^{i}$ is the Kronecker delta.

Let $L_{1}$ be the $n \times n$-matrix whose $j$-th column is $a_{j}$, and let $L_{2}$ be the $n \times n$-matrix whose $i$-th column is $b_{i}$, then $L_{1}^{-1}=L_{2}^{T}$, $L_{2}^{-1}=L_{1}^{T}$. Introduce $\Lambda=\operatorname{diag}\left(\lambda_{1}, \lambda_{2}, \ldots, \lambda_{n}\right)$. Then $L_{2} \Lambda=M_{2} L_{1}$ and $M_{1} L_{2}=L_{1} \Lambda$, which implies

$$
M_{1}=L_{1} \Lambda L_{1}^{T}, M_{2}=L_{2} \Lambda L_{2}^{T}
$$

Introducing the diagonal matrix $\tilde{\Lambda}=\operatorname{diag}(\Lambda,-\Lambda)$ and using Eqs. (13), we finally obtain

$$
M=L \tilde{\Lambda} L^{-1}
$$

where

$$
L=\frac{1}{\sqrt{2}}\left(\begin{array}{cc}
L_{1} & L_{1} \\
L_{2} & -L_{2}
\end{array}\right), L^{-1}=\frac{1}{\sqrt{2}}\left(\begin{array}{cc}
L_{2}^{T} & L_{1}^{T} \\
L_{2}^{T} & -L_{1}^{T}
\end{array}\right)
$$


In our case the explicit formulas for $\lambda_{j}, a_{j}, b_{j}$, are:

System 1. There are three modes: fast compressional wave $\left(\lambda_{1}^{(1)}\right)$, Biot slow wave $\left(\lambda_{2}^{(1)}\right)$ and vertical shear wave $\left(\lambda_{3}^{(1)}\right)$.

$$
\begin{aligned}
& \left(\lambda_{j}^{(1)}\right)^{2}=-\gamma^{2}+\beta\left(c \rho_{f}-\frac{m \rho}{2}+\frac{(\lambda+2 \mu)\left(d-i \omega \rho_{\omega}\right)}{2 i \omega}\right) \pm \\
& \pm \frac{\beta}{2} \sqrt{\left(m \rho+\frac{(\lambda+2 \mu)\left(d-i \omega \rho_{\omega}\right)}{i \omega}\right)^{2}-4\left(m \rho_{f}+c \frac{d-i \omega \rho_{\omega}}{i \omega}\right)\left(c \rho-(\lambda+2 \mu) \rho_{f}\right)} \\
& j=1,2 \text {, with (+) for } j=1 \text { and (-) for } j=2, \text { and }\left(\lambda_{3}^{(1)}\right)^{2}=-\gamma^{2}+\mu^{-1}\left(\rho+\frac{i \omega \rho_{f}^{2}}{d-i \omega \rho_{\omega}}\right) \\
& a_{j}^{(1)}=\bar{a}_{j}\left(-1,2 \mu \gamma, \xi_{j}\right)^{T}, j=1,2, a_{3}^{(1)}=\frac{\bar{a}_{3}}{\lambda_{3}^{(1)}}\left(\gamma, \mu\left(\lambda_{3}^{(1)}\right)^{2}-\mu \gamma^{2},-\frac{i \omega \gamma \rho_{f}}{d-i \omega \rho_{\omega}}\right)^{T} \\
& b_{j}^{(1)}=\frac{\bar{a}_{j}}{\lambda_{j}^{(1)}}\left(2 \mu \gamma^{2}-\rho-\rho_{f} \xi_{j}, \gamma, \rho_{f}-\xi_{j} \frac{d-i \omega \rho_{\omega}}{i \omega}\right)^{T}, j=1,2, b_{3}^{(1)}=\bar{a}_{3}(2 \mu \gamma, 1,0)^{T}
\end{aligned}
$$

where

$$
\begin{aligned}
& \xi_{j}=\frac{c \rho-(\lambda+2 \mu) \rho_{f}}{\frac{\left(\lambda_{j}^{(1)}\right)^{2}+\gamma^{2}}{\beta}-c \rho_{f}+i(\lambda+2 \mu) \frac{d-i \omega \rho_{\omega}}{i \omega}}, j=1,2, \\
& \bar{a}_{j}=\sqrt{\frac{\lambda_{j}^{(1)}}{\rho+2 \rho_{f} \xi_{j}+i \xi_{j}^{2} \frac{d-i \omega \rho_{\omega}}{i \omega}}}, j=1,2, \bar{a}_{3}=\sqrt{\frac{\lambda_{3}^{(1)}}{\mu\left(\lambda_{3}^{(1)}\right)^{2}+\mu \gamma^{2}}} .
\end{aligned}
$$

System 2. There is the horizontal shear wave $\left(\lambda^{(2)}\right)$ only.

$$
\left(\lambda^{(2)}\right)^{2}=-\gamma^{2}+\mu^{-1}\left(\rho+\frac{i \omega \rho_{f}^{2}}{d-i \omega \rho_{\omega}}\right), a^{(2)}=\sqrt{\frac{1}{\mu \lambda(2)}}, b^{(2)}=\sqrt{\mu \lambda(2)} .
$$

\section{Reflection and transmission matrices}

Firstly, we consider a homogeneous source-free region of space. Dropping ${ }^{(m)}$ we have a $2 n$-dimensional system of the form (7) with $M$ constant and $S=0$. Let

$$
\Phi=L \Psi \text { and } \Psi=(U, D)^{T},
$$

where $U, D$ are $n$-vectors. Inserting Eqs. (15) into Eqs. (7) and using Eq. (14) we arrive at

$$
\frac{d}{d z} \Psi=-i \omega \tilde{\Lambda} \Psi
$$

Then

$$
\Psi(z)=\left(e^{-i \omega \Lambda\left(z-z_{0}\right)} U\left(z_{0}\right), e^{i \omega \Lambda\left(z-z_{0}\right)} D\left(z_{0}\right)\right)^{T},
$$

where $z_{0}$ is a fixed point in the same source-free region. The vectors $U, D$ characterize up-going $(U)$ and down-going $(D)$ waves. Next, consider an interface at $z=\bar{z}$, where the material parameters vary discontinuously across $\bar{z}$. We denote by ${ }^{ \pm}$quantities evaluated at $\bar{z}^{ \pm}=\bar{z} \pm 0$. Since $\Phi$ is continuous across $\bar{z}$, we obtain

$$
\Psi^{+}=J \Psi^{-}, \Psi^{-}=J^{-1} \Psi^{+},
$$

where the jump matrix is

$$
J=\left(L^{+}\right)^{-1} L^{-}=\left(\begin{array}{cc}
J_{A} & J_{B} \\
J_{B} & J_{A}
\end{array}\right), J^{-1}=\left(\begin{array}{cc}
J_{A}^{T} & -J_{B}^{T} \\
-J_{B}^{T} & J_{A}^{T}
\end{array}\right)
$$

and $J_{A}, J_{B}$ are the $n \times n$-matrices

$$
J_{A}=\frac{1}{2}\left[\left(L_{2}^{+}\right)^{T} L_{1}^{-}+\left(L_{1}^{+}\right)^{T} L_{2}^{-}\right], J_{B}=\frac{1}{2}\left[\left(L_{2}^{+}\right)^{T} L_{1}^{-}-\left(L_{1}^{+}\right)^{T} L_{2}^{-}\right] .
$$


Next, we consider a stack of layers $0<z_{1}<\cdots<z_{N}<\infty$. We denote by subscript $j$ a quantity at interface $z=z_{j}$, with superscripts ${ }^{ \pm}$as before. Then

$$
\left(U_{N}^{-}, D_{N}^{-}\right)^{T}=J_{N}^{-1}\left(0, D_{N}^{+}\right)^{T},
$$

where we have used that there is no up-going wave below the last interface at $z=z_{N}$. So, we obtain

$$
U_{N}^{-}=\Gamma_{N} D_{N}^{-}, D_{N}^{+}=T_{N} D_{N}^{-},
$$

where

$$
\Gamma_{N}=-J_{B, N}^{T}\left(J_{A, N}^{T}\right)^{-1}, T_{N}=\left(J_{A, N}^{T}\right)^{-1} .
$$

Here $\Gamma_{N}$ is the reflection matrix and $T_{N}$ is the transmission matrix from the last interface $z=z_{N}$.

Let $j<N$ and $\triangle z_{j}=z_{j+1}-z_{j}, j=0,1, \ldots, N-1$, is the layer thickness. Then by jumping across the layer boundary and using Eqs. (16) and (17) we obtain

$$
U_{j}^{-}=J_{A, j}^{T} e^{i \omega \Lambda_{j} \Delta z_{j}} U_{j+1}^{-}-J_{B, j}^{T} e^{-i \omega \Lambda_{j} \Delta z_{j}} D_{j+1}^{-}, D_{j}^{-}=-J_{B, j}^{T} e^{i \omega \Lambda_{j} \Delta z_{j}} U_{j+1}^{-}+J_{A, j}^{T} e^{-i \omega \Lambda_{j} \Delta z_{j}} D_{j+1}^{-} .
$$

Define reflection and transmission matrices $\Gamma_{j}, T_{j}$ by the relations that for any incident wave $D_{j}^{-}$at the top of stack of layers underlying $z=z_{j}$

$$
U_{j}^{-}=\Gamma_{j} D_{j}^{-}, D_{j}^{+}=T_{j} D_{j}^{-} .
$$

Therefore $\Gamma_{j}$ computes the reflected wave from the stack and $T_{j}$ computes the transmitted wave below the stack, when the incident wave is known. From Eqs. (19) and (20) we obtain by induction

$$
\Gamma_{j}=\left(J_{A, j}^{T} \tilde{\Gamma}_{j+1}-J_{B, j}^{T}\right)\left(-J_{B, j}^{T} \tilde{\Gamma}_{j+1}+J_{A, j}^{T}\right)^{-1}, T_{j}=T_{j+1} e^{i \omega \Lambda_{j} \Delta z_{j}}\left(-J_{B, j}^{T} \tilde{\Gamma}_{j+1}+J_{A, j}^{T}\right)^{-1},
$$

where $\tilde{\Gamma}_{j+1}=e^{i \omega \Lambda_{j} \Delta z_{j}} \Gamma_{j+1} e^{i \omega \Lambda_{j} \Delta z_{j}}$. Again, by induction it can be shown that $\Gamma_{j}$ is symmetric. Thus all the reflection and transmission matrices can be calculated by Eqs. (21), starting with Eqs. (18).

\section{Sources and boundary conditions}

Consider a $2 n$-dimensional system of the form (7) with ${ }^{(m)}$ omitted. Let the source be of the form

$$
S=S_{0} \delta\left(z-z_{s}\right)+S_{1} \delta^{\prime}\left(z-z_{s}\right)
$$

with $S_{0}, S_{1}$ independent of $z$. Here $\delta$ is the Dirac function. Define $n$-vectors $S_{A}, S_{B}$ by the following formula

$$
\left(S_{A}, S_{B}\right)^{T}=i \omega M S_{1}-S_{0}
$$

Applying the standard procedure we obtain the following jump condition across the source

$$
\Phi\left(z_{s}^{-}\right)=\Phi\left(z_{s}^{+}\right)+\left(S_{A}, S_{B}\right)^{T} .
$$

Inserting a fictitious layer boundary at $z=z_{s}^{+}$, we compute the reflection matrix $\Gamma_{s} \equiv \Gamma\left(z_{s}^{+}\right)$from the top of this layer. Note that at $z_{s}^{+}$, $J_{A}=I, J_{B}=0$, since the material properties do not change at $z_{s}$. Then the up-going wave $U_{s} \equiv U_{s}\left(z_{s}^{+}\right)$is related to the down-going wave $D_{s} \equiv D_{s}\left(z_{s}^{+}\right)$there by Eqs. (20). Then we have

$$
\Psi\left(z_{s}^{+}\right)=\left(\Gamma_{s} D_{s}, D_{s}\right)^{T}
$$

Using Eqs. (15), (24) and (25) we obtain

$$
\Psi\left(z_{s}^{-}\right)=\left(\Gamma_{s} D_{s}, D_{s}\right)^{T}+\frac{1}{\sqrt{2}}\left(L_{2}^{T} S_{A}+L_{1}^{T} S_{B}, L_{2}^{T} S_{A}-L_{1}^{T} S_{B}\right)^{T} .
$$


This expression may now propagated upwards through layers, using Eq. (16) and jumped upwards across layers boundaries using Eqs. (17) until we reach the free surface at $z=0^{+}$. Then the $n$ boundary conditions at $z=0$ can be used to find the $n$ unknowns $D_{s}$. Consider now one particular case when $z_{s} \in\left(0, z_{1}\right)$. In this case

$$
\Psi\left(0^{+}\right)=\left(e^{i \omega \Lambda_{s} z_{s}} \Gamma_{s} D_{s}, e^{-i \omega \Lambda_{s_{s}}} D_{s}\right)^{T}+\frac{1}{\sqrt{2}}\left(e^{i \omega \Lambda_{s} z_{s}}\left(L_{2}^{T} S_{A}+L_{1}^{T} S_{B}\right), e^{-i \omega \Lambda_{s} z_{s}}\left(L_{2}^{T} S_{A}-L_{1}^{T} S_{B}\right)\right)^{T} .
$$

We next write

$$
\Phi\left(0^{+}\right)=\left(G_{A} \Phi_{0}, G_{B} \Phi_{0}\right)^{T},
$$

where $\Phi_{0}$ is an $n$-vector of unknowns at $z=0$ and $G_{A}, G_{B}$ are $n \times n$ matrices. For System 1 , let

$$
\Phi_{0}^{(1)}=\left(\tilde{v}_{3},-\tilde{q}_{3}, \tilde{v}_{1}\right)_{z=0^{+}}^{T}, G_{A}^{(1)}=\left(\begin{array}{ccc}
1 & 0 & 0 \\
0 & 0 & 0 \\
0 & 1 & 0
\end{array}\right), G_{B}^{(1)}=\left(\begin{array}{ccc}
0 & 0 & 0 \\
0 & 0 & 1 \\
0 & 0 & 0
\end{array}\right) .
$$

We can check that Eq. (27) holds for System 1 with the boundary conditions given by Eqs. (12). For System 2, let

$$
\Phi_{0}^{(2)}=\tilde{v}_{2}\left(0^{+}\right), G_{A}^{(2)}=1, G_{B}^{(2)}=0 .
$$

It may be checked that Eq. (27) holds for System 2 with the boundary conditions given by Eqs. (12).

Using Eqs. (15), (26) and (27) we obtain

$$
\begin{aligned}
& \Phi_{0}=\left(e^{i \omega \Lambda z_{s}} \Gamma_{s} e^{i \omega \Lambda z_{s}}\left(L_{2}^{T} G_{A}-L_{1}^{T} G_{B}\right)-\left(L_{2}^{T} G_{A}+L_{1}^{T} G_{B}\right)\right)^{-1} \times \\
& \times e^{i \omega \Lambda z_{s}}\left(\Gamma_{s}\left(L_{2}^{T} S_{A}-L_{1}^{T} S_{B}\right)-\left(L_{2}^{T} S_{A}+L_{1}^{T} S_{B}\right)\right), \\
& D_{s}=\frac{1}{\sqrt{2}} e^{i \omega \Lambda z_{s}}\left(L_{2}^{T} G_{A}-L_{1}^{T} G_{B}\right) \Phi_{0}-\frac{1}{\sqrt{2}}\left(L_{2}^{T} S_{A}-L_{1}^{T} S_{B}\right) .
\end{aligned}
$$

In particular, when the source is situated just below the surface we get

$$
\Phi_{0}=\left(\left(\Gamma_{s}-I\right) L_{2}^{T} G_{A}-\left(\Gamma_{s}+I\right) L_{1}^{T} G_{B}\right)^{-1} \times\left(\left(\Gamma_{s}-I\right) L_{2}^{T} S_{A}-\left(\Gamma_{s}+I\right) L_{1}^{T} S_{B}\right) \text { as } z_{s} \rightarrow 0^{+} .
$$

$\Phi_{0}$ defines all of $\Phi$ at the free surface, and $D_{s}, U_{s}=\Gamma_{s} D_{s}$ give all of $\Phi$ just below the source. Now we are able to compute $\Phi$ in any $z \in \mathbb{R}_{+}$by propagating through the layers using Eqs. (16) and (17).

Remark2. Propagation of an upward-going wave in the downward direction will be unstable numerically using Eq. (16), because the complex exponentials grow rather than decay with distance. Therefore, numerically one has to obtain $U$ from $D$ using the reflection or transmission matrices.

Inverting Eqs. (5), we can calculate the hat (^) variables, i.e.,

$$
\hat{v}=\Omega^{T} \tilde{v}, \hat{q}=\Omega^{T} \tilde{q}, \hat{\tau}=\Omega^{T} \tilde{\tau} \Omega, \hat{p}=\tilde{p} .
$$

To get the solution in real space we need to apply the inverse Fourier transform $F_{x_{1} x_{2}}^{-1}$. The matrices for Systems 1 and 2 depend only on the magnitude $k$. However, factors $k_{1}$ and $k_{2}$ are introduced by Eq. (6) and possibly by the directionality of the source. For any function $\hat{h}(k)$ let

$$
\mathscr{T}_{j_{1}, j_{2}}(\hat{h}) \equiv F_{x_{1} x_{2}}^{-1}\left(k_{1}^{j_{1}} k_{2}^{j_{2}} \hat{h}(k)\right)=(-i)^{j_{1}+j_{2}} \partial_{x_{1}}^{j_{1}} \partial_{x_{2}}^{j_{2}} F_{x_{1} x_{2}}^{-1}(\hat{h}(k)) .
$$

We can compute these quantities as Hankel transforms in the cylindrical coordinates $r, \theta, z$. Define

$$
B_{j_{1}, j_{2}}(\hat{h})=\frac{1}{2 \pi} \int_{0}^{\infty} k^{j_{1}} J_{j_{2}}(k r) \hat{h}(k) d k,
$$


where $J_{j_{2}}$ is the Bessel function and $j_{1}, j_{2}$ are nonnegative integers. Then, in particular,

$$
\begin{aligned}
& \mathscr{T}_{0,0}=B_{1,0}, \mathscr{T}_{1,0}=i \cos \theta B_{2,1}, \mathscr{T}_{0,1}=i \sin \theta B_{2,1}, \mathscr{T}_{1,1}=\sin \theta \cos \theta\left(B_{3,0}-\frac{2}{r} B_{2,1}\right), \\
& \mathscr{T}_{2,0}=\cos ^{2} \theta B_{3,0}-\frac{\cos 2 \theta}{r} B_{2,1}, \mathscr{T}_{0,2}=\sin ^{2} \theta B_{3,0}+\frac{\cos 2 \theta}{r} B_{2,1} .
\end{aligned}
$$

\section{Dynamite source}

A dynamite source imposed on the solid and the fluid can be defined in the following form

$$
f(x)=g(x)=-s(\omega) \nabla \delta\left(x-x_{s}\right)
$$

where $x_{s}=\left(0,0, z_{s}\right)^{T}$ is the source position and $s(\omega)$ is the spectrum of the seismic moment. Applying the Fourier transform $F_{x_{1} x_{2}}$ we obtain

$$
\hat{f}=\hat{g}=-s(\omega)\left(i k_{1} \boldsymbol{\delta}\left(z-z_{s}\right), i k_{2} \boldsymbol{\delta}\left(z-z_{s}\right), \boldsymbol{\delta}^{\prime}\left(z-z_{s}\right)\right)^{T} .
$$

The rotation by $\Omega$ yields

$$
\tilde{f}=\tilde{g}=-s(\omega)\left(i k \delta\left(z-z_{s}\right), 0, \delta^{\prime}\left(z-z_{s}\right)\right)^{T} .
$$

Substitution of Eq. (32) into Eqs. (9) gives the source for System 1 in the form (22) with

$$
S_{0}^{(1)}=s(\omega)\left(0, i k-\frac{\omega \rho_{f}}{d-i \omega \rho_{\omega}}, \frac{k^{2}}{d-i \omega \rho_{\omega}}, 0,0,0\right)^{T}, S_{1}^{(1)}=s(\omega)(0,0,0,1,0,-1)^{T} .
$$

Substitution of Eq. (32) into Eqs. (10) shows that $S^{(2)}$ is zero, then $\tilde{v}_{2}, \tilde{\tau}_{23}$ associated with System 2 are zero too. This is to be expected result because System 2 is related to SH-waves, which are not excited by the dynamic source. Substitution of Eqs. (33) into (23) gives

$$
S_{A}^{(1)}=i \beta s(w)(\omega(c-m), 2 k \mu(m-c), \omega(\lambda+2 \mu-c))^{T}, S_{B}^{(1)}=(0,0,0)^{T} .
$$

Eqs. (34) may be used in Eqs. (28) or (29) for a shallow source, to obtain all the tilde ( ${ }^{\sim}$ ) functions.

To invert the rotation $\Omega$, using Eqs. (30), note that from Eqs. (11) and the vanishing of System 2, $\tilde{v}_{2}, \tilde{q}_{2}, \tilde{\tau}_{12}, \tilde{\tau}_{23}$ are identically zero. All the remaining tilde functions depend of $k$ only and can be calculated by the following formulas

$$
\begin{aligned}
& \hat{v}_{1}=\frac{k_{1}}{k} \tilde{v}_{1}, \hat{v}_{2}=\frac{k_{2}}{k} \tilde{v}_{1}, \hat{v}_{3}=\tilde{v}_{3}, \hat{q}_{1}=\frac{k_{1}}{k} \tilde{q}_{1}, \hat{q}_{2}=\frac{k_{2}}{k} \tilde{q}_{1}, \hat{q}_{3}=\tilde{q}_{3}, \\
& \hat{\tau}_{11}=\frac{k_{1}^{2} \tilde{\tau}_{11}+k_{2}^{2} \tilde{\tau}_{22}}{k^{2}}, \hat{\tau}_{12}=\frac{k_{1} k_{2}\left(\tilde{\tau}_{11}-\tilde{\tau}_{22}\right)}{k^{2}}, \hat{\tau}_{22}=\frac{k_{2}^{2} \tilde{\tau}_{11}+k_{1}^{2} \tilde{\tau}_{22}}{k^{2}}, \quad \hat{\tau}_{13}=\frac{k_{1} \tilde{\tau}_{13}}{k}, \hat{\tau}_{23}=\frac{k_{2} \tilde{\tau}_{13}}{k}, \hat{\tau}_{33}=\tilde{\tau}_{33} .
\end{aligned}
$$

Then the Fourier transform $F_{x_{1} x_{2}}$ can be inverted in cylindrical coordinates $(r, \theta, z)$ using Eqs. (31) to obtain the solid and fluid velocities

$$
v=\left(i B_{1,1}\left(\tilde{v}_{1}\right)\right) e_{r}+\left(B_{1,0}\left(\tilde{v}_{3}\right)\right) e_{z}, q=\left(i B_{1,1}\left(\tilde{q}_{1}\right)\right) e_{r}+\left(B_{1,0}\left(\tilde{q}_{3}\right)\right) e_{z}
$$

and the stress tensor components

$$
\begin{aligned}
& \tau_{11}=\mathscr{T}_{2,0}\left(k^{-2} \tilde{\tau}_{11}\right)+\mathscr{T}_{0,2}\left(k^{-2} \tilde{\tau}_{22}\right), \tau_{12}=\mathscr{T}_{1,1}\left(k^{-2}\left(\tilde{\tau}_{11}-\tilde{\tau}_{22}\right)\right), \\
& \tau_{22}=\mathscr{T}_{0,2}\left(k^{-2} \tilde{\tau}_{11}\right)+\mathscr{T}_{2,0}\left(k^{-2} \tilde{\tau}_{22}\right), \tau_{13}=\mathscr{T}_{1,0}\left(k^{-1} \tilde{\tau}_{13}\right), \tau_{23}=\mathscr{T}_{0,1}\left(k^{-1} \tilde{\tau}_{13}\right), \tau_{33}=\mathscr{T}_{0,0}\left(\tilde{\tau}_{33}\right) .
\end{aligned}
$$

These stresses may now be computed in cylindrical coordinates from Eqs. (31) using the Hankel transforms of the appropriate tilde functions. 


\section{Vertical source}

Consider a vertical point force acting on the free surface $z=0$, i.e.,

$$
f(x)=g(x)=(0,0,1)^{T} s(\omega) \delta\left(x_{1}\right) \delta\left(x_{2}\right) \delta\left(z-z_{s}\right),
$$

where $z_{s} \rightarrow 0^{+}$puts the force on the free surface. This models hammer, weight drop, and vibroseis sources. Applying the Fourier transform $F_{x_{1} x_{2}}$ and rotation $\Omega$ we arrive at

$$
\tilde{f}=\tilde{g}=\hat{f}=\hat{g}=(0,0,1)^{T} s(\omega) \delta\left(z-z_{s}\right) .
$$

Substitution of Eqs. (38) into Eqs. (9), (10) yields the source for Systems 1 and 2 in the form

$$
S^{(1)}=(0,0,0,-1,0,1)^{T} s(\omega) \delta\left(z-z_{s}\right), S^{(2)}=(0,0)^{T} .
$$

Thus, all variables in System 2 are zero, as it was in the case of the dynamite source. From Eqs. (22), (23) and (39) we obtain

$$
S_{A}^{(1)}=(0,0,0)^{T}, S_{B}^{(1)}=(1,0,-1)^{T} s(\omega) .
$$

Now all the tilde variables at the free surface may be computed from Eqs. (29) as $z_{s} \rightarrow 0^{+}$and propagated anywhere else in space. Note that $S_{A}^{(1)}, S_{B}^{(1)}$ are independent of $k_{1}, k_{2}$, so that the tilde variables depend only on $k$ and not on wavenumber direction. Therefore, similar to dynamite we can transform to the hat variables using Eqs. (35) and transform back to the spatial coordinates using Eqs. (36) and (37).

\section{RESULTS}

A one-dimensional analysis of horizontally stratified geological models composed of three homogeneous and isotropic layers is considered.

\section{Dispersion and attenuation analysis}

In a recent paper, Blanc (2013) derived the dispersion relationship between frequency and wave number. Thus, given the wave numbers $k_{p f}(\omega), k_{p s}(\omega)$, where $k_{p f}$ is the wave number for the fast $P$-wave, $k_{p s}$ is the wave number for the slow $P$-wave, we obtain the phase velocities

$$
c_{p f}(\omega)=\frac{\omega}{\operatorname{Re}\left[k_{p f}(\omega)\right]}, c_{p s}(\omega)=\frac{\omega}{\operatorname{Re}\left[k_{p s}(\omega)\right]},
$$

where $0<c_{p s}<c_{p f}$, and attenuations

$$
\alpha_{p f}=\operatorname{Im}\left[k_{p f}(\omega)\right], \alpha_{p s}=\operatorname{Im}\left[k_{p s}(\omega)\right] .
$$

Figures 1 and 2 show the dispersion and attenuation curves corresponding to the Biot and Biot-JKD models (fast and slow $P$-waves only) for four distinct experiments: $100 \%$ porous medium saturated with (1) water; (2) light oil $36^{\circ} \mathrm{API}$; (3) $24.7^{\circ} \mathrm{API}$ medium oil and (4) heavy oil $14.8^{\circ} \mathrm{API}$. The physical properties of the porous media and fluids were taken from the works of Blanc (2013) and Al-Besharah et al. (1987), and are present in Table 1.

\section{Numerical simulation}

The physical properties of each layer, used in the numerical simulations, are listed in Table 2.

In order to perform the elastic wave propagation simulations, a source was used, represented by a truncated sine wave function defined by the following formula, see Blanc (2013):

$$
H(t)=\left\{\begin{array}{ll}
\sin \left(\omega_{d} t\right)-\frac{21}{32} \sin \left(2 \omega_{d} t\right)+\frac{63}{768} \sin \left(4 \omega_{d} t\right)-\frac{1}{512} \sin \left(8 \omega_{d} t\right), & \text { if } 0 \leq t \leq f_{d}^{-1} \\
0, & \text { if } t>f_{d}^{-1}
\end{array},\right.
$$

where $\omega_{d}=2 \pi f_{d}$. The shape of $H(t)$ with the dominant frequency $20 \mathrm{~Hz}$ is shown in Figure 3 . 


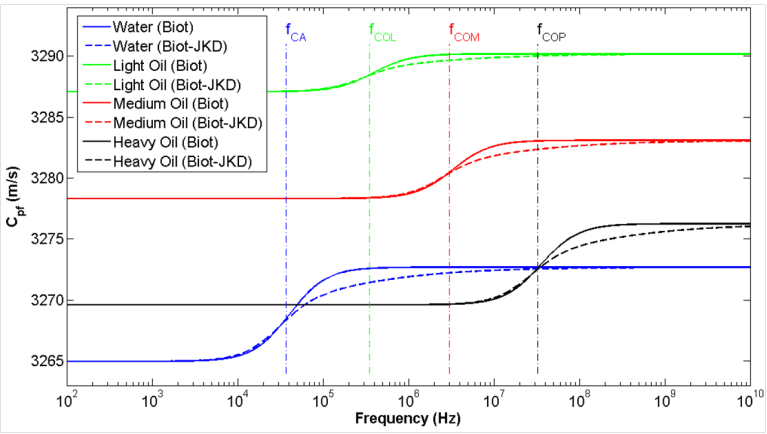

(a)

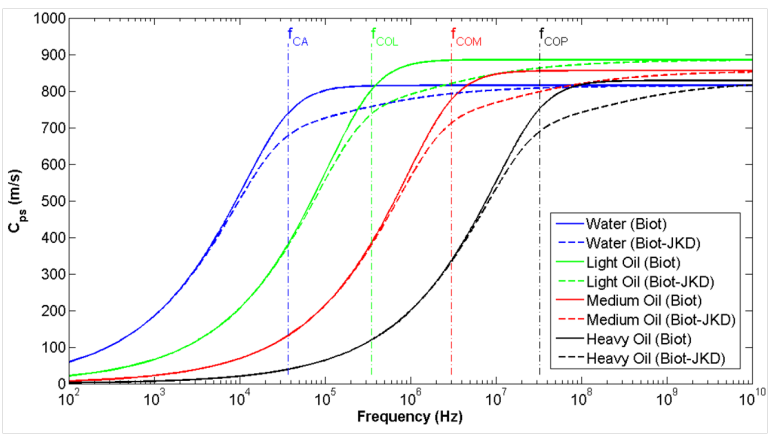

(b)

Figure 1 - Phase velocity curves: fast (a) and slow (b) waves.

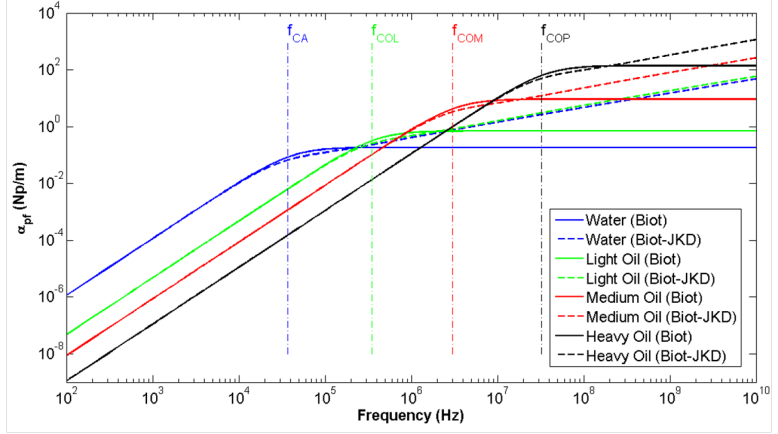

(a)

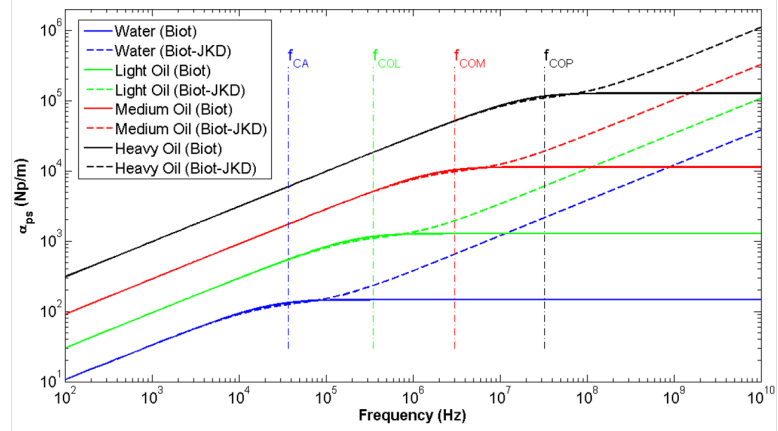

(b)

Figure 2 - Attenuation curves: fast (a) and slow (b) waves.

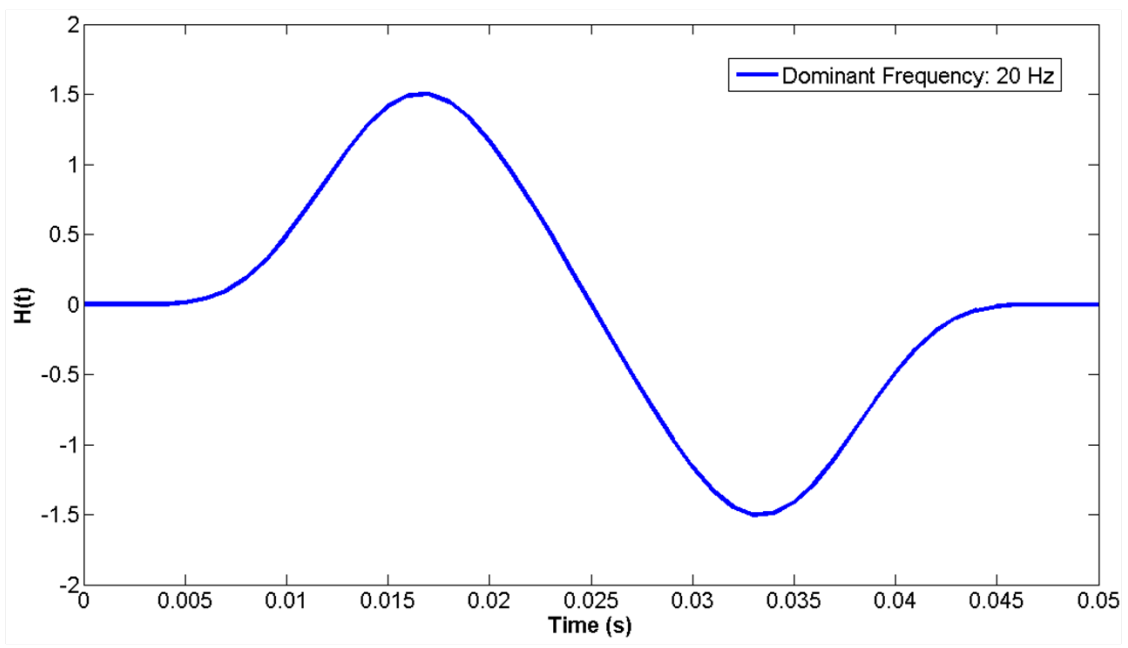

Figure 3 - Shape of $H(t)$. 
Table 1 - Physical properties used for dispersion and attenuation analysis.

\begin{tabular}{|l|c|c|c|}
\hline Property & Symbol & Unity & Values \\
\hline Density of water & $\rho_{w}$ & $\mathrm{~kg} / \mathrm{m}^{3}$ & 1000 \\
\hline Density of light oil & $\rho_{o l}$ & $\mathrm{~kg} / \mathrm{m}^{3}$ & 844.8 \\
\hline Density of medium oil & $\rho_{o m}$ & $\mathrm{~kg} / \mathrm{m}^{3}$ & 905.9 \\
\hline Density of heavy oil & $\rho_{\text {oh }}$ & $\mathrm{kg} / \mathrm{m}^{3}$ & 967.2 \\
\hline Viscosity of water & $\eta_{w}$ & $\mathrm{~Pa} . \mathrm{S}$ & $10^{-3}$ \\
\hline Viscosity of light oil & $\eta_{o l}$ & $\mathrm{~Pa} . \mathrm{S}$ & $8.03 \times 10^{-3}$ \\
\hline Viscosity of medium oil & $\eta_{o m}$ & $\mathrm{~Pa} . \mathrm{S}$ & $73.79 \times 10^{-3}$ \\
\hline Viscosity of heavy oil & $\eta_{o h}$ & $\mathrm{~Pa} . \mathrm{S}$ & $853.78 \times 10^{-3}$ \\
\hline Density of solid & $\rho_{s}$ & $\mathrm{~kg} / \mathrm{m}^{3}$ & 2644 \\
\hline Shear modulus & $\mu$ & $\mathrm{Pa}$ & $7.04 \times 10^{9}$ \\
\hline Porosity & $\phi$ & - & 0.2 \\
\hline Tortuosity & $a$ & - & 2.04 \\
\hline Permeability & $\kappa_{0}$ & $\mathrm{~m}^{2}$ & $3.6 \times 10^{-13}$ \\
\hline Lamé parameter & $\lambda$ & $\mathrm{Pa}$ & $1.06 \times 10^{10}$ \\
\hline Biot modulus & $m$ & $\mathrm{~Pa}$ & $9.7 \times 10^{9}$ \\
\hline Biot modulus & $\Lambda$ & $\mathrm{m}$ & $5.88 \times 10^{-6}$ \\
\hline Viscous characteristic length & $\Lambda$ & & \\
\hline
\end{tabular}

Table 2 - Physical properties used for simulation of poroelastic waves.

\begin{tabular}{|l|c|c|c|c|}
\hline Property & Symbol & Unity & Layers 1\&3 & Layer 2 \\
\hline Density of fluid & $\rho_{w}$ & $\mathrm{~kg} / \mathrm{m}^{3}$ & 1040 & 1000 \\
\hline Viscosity of fluid & $\eta_{w}$ & $\mathrm{~Pa} . \mathrm{S}$ & $10^{-3}$ & $10^{-3}$ \\
\hline Density of solid & $\rho_{s}$ & $\mathrm{~kg} / \mathrm{m}^{3}$ & 2650 & 2644 \\
\hline Shear modulus & $\mu$ & $\mathrm{Pa}$ & $1.85 \times 10^{9}$ & $7.04 \times 10^{9}$ \\
\hline Porosity & $\phi$ & - & 0.3 & 0.2 \\
\hline Tortuosity & $a$ & - & 2 & 2.04 \\
\hline Permeability & $\kappa_{0}$ & $\mathrm{~m}^{2}$ & $10^{-12}$ & $3.6 \times 10^{-13}$ \\
\hline Lamé parameter & $\lambda$ & $\mathrm{Pa}$ & $8.4 \times 10^{9}$ & $1.06 \times 10^{10}$ \\
\hline Biot modulus & $m$ & $\mathrm{~Pa}$ & $7.05 \times 10^{9}$ & $9.7 \times 10^{9}$ \\
\hline Biot modulus & $c$ & - & 0.88 & 0.72 \\
\hline Viscous characteristic length & $\Lambda$ & $\mathrm{m}$ & $7.3 \times 10^{-6}$ & $5.88 \times 10^{-6}$ \\
\hline
\end{tabular}


The results of the simulations are presented separately in relation to the frequency domain they belong to: low frequencies (reservoir scale) and high frequencies (laboratory scale). For the low frequency domain a source with a dominant frequency of $20 \mathrm{~Hz}$ was used. It was considered that both the source and the receiver are located on the free surface, as shown in Figure 4(a).

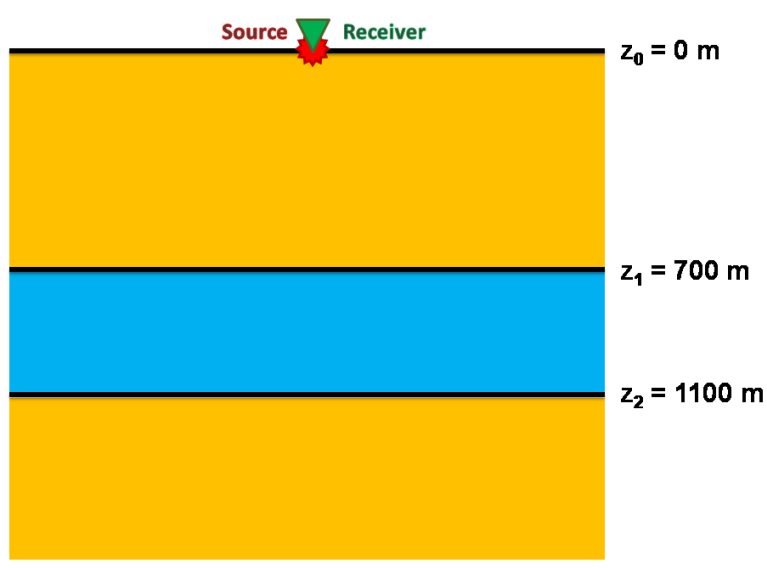

(a)

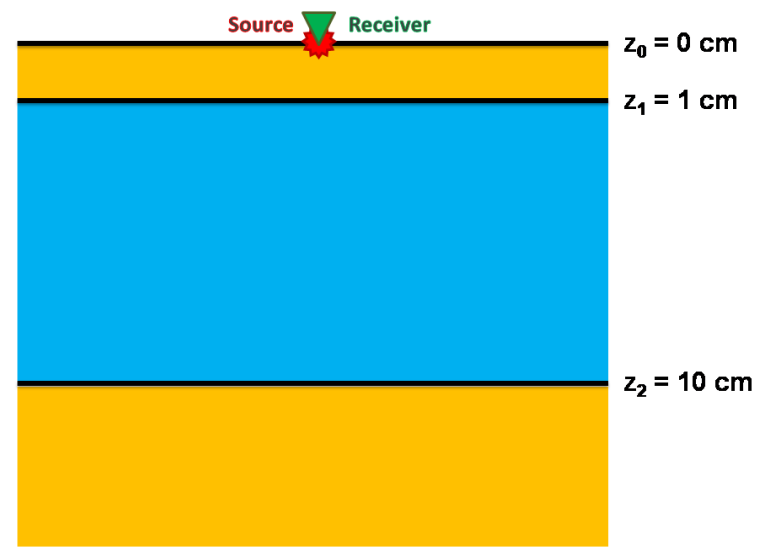

(b)

Figure 4 - Physical models used in numerical experiments: reservoir (a) and laboratory (b) scales.

Figure 5(a) shows the result obtained in this simulation, where the absolute displacement velocity of the solid phase was plotted for both the Biot model (blue curve) and the Biot-JKD model (red curve). The numbered arrows indicate the arrival times of the events detected in the simulation. For low frequencies the medium does not support the slow wave, which becomes diffusive. Moreover, since a one-dimensional analysis was performed, there is no presence of the shear wave, so all the observed events refer to the propagation of the fast compressional wave in the poroelastic medium. Figure $5(\mathrm{~b})$ presents the same result of Figure 5(a), but with a zoom in the vertical axis for better visualization of the events that have smaller amplitudes.

For the domain of the high frequencies, a source with a dominant frequency of $200 \mathrm{kHz}$ was used. And, likewise, both the source and the receiver are located on the free surface, see Figure 4(b). For high frequencies, besides the presence of the fast wave, we can observe the slow wave propagating in the poroelastic medium, see Figure 6 . Another phenomenon seen in this simulation was the presence of the the converted fast-slow $P$-wave, where the elastic wave converts its propagation mode by refracting or reflecting at a discontinuity interface between two distinct poroelastic media, as observed by Bouzidi (2013).

\section{DISCUSSION}

By dispersion and attenuation analysis of the waves propagating in a 1D poroelastic medium (Figs. 1 and 2) it was found that for low frequencies and for frequencies much larger than the critical frequency, the denser the fluid, the lower the velocity of phase of the rapid wave. In addition, the more viscous the fluid, the slower the attenuation of the slow wave, both for the Biot model and the Biot-JKD model and for low frequencies, the more viscous the fluid, the lower the fast wave attenuation, even at very high frequencies, the opposite occurs: the more viscous the fluid, the greater the attenuation of the fast wave, for both models.

Based on the theory of poroelasticity, we sought to analyze the results of the simulations. The first analysis was the confirmation that the arrival times of the seismic events, for the simulations in the two domains of low and high frequencies, were correct. For this purpose we used the following formula $t_{m}=$ $\delta z / c_{p}$, where $t_{m}$ indicates the transit time of the compressional wave within the layer under analysis, $\delta z$ represents the distance traveled by the wave within the layer $m$ and $c_{p}$ is the wave propagation speed in the layer, whether the wave is fast or slow.

Then, it was observed that the results obtained for the high frequency domain (Fig. 6) presented an amplitude difference for the seismic events related to the slow wave between the two models used, of Biot and Biot-JKD. The explanation for such phenomenon is found in the dispersion and attenuation analysis of the waves propagating through a $1 \mathrm{D}$ poroelastic medium (Figs. 1 and 2), more specifically in Figure 2(b), where it can be observed that the attenuation for the Biot-JKD model is higher than for the Biot model in the high frequency domain. However, there were no differences in amplitude between the Biot and Biot-JKD models in the low frequency range, which was already expected theoretically, given that for this domain the 

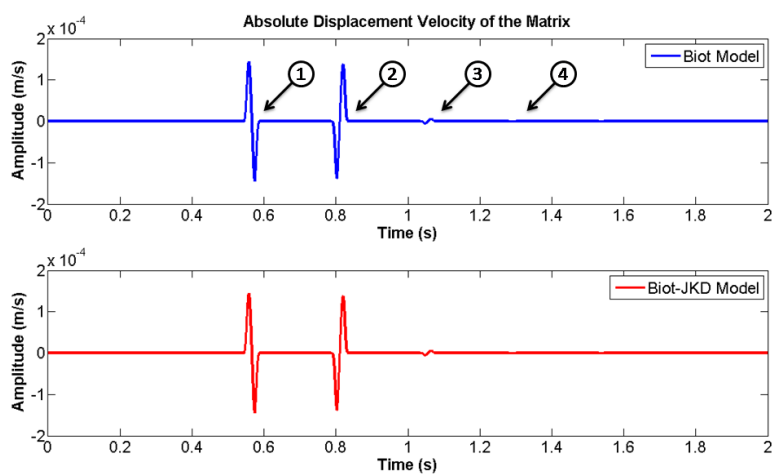

(a)
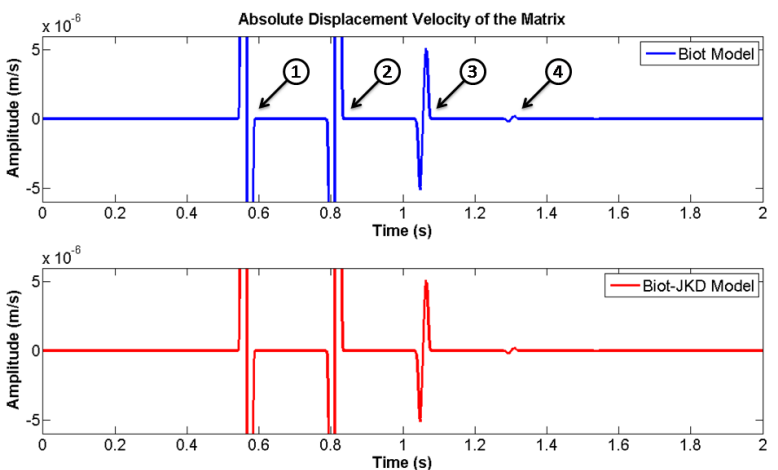

(b)

Figure $\mathbf{5}$ - Reservoir scale results (a) and the same with zoom in the vertical axis (b). The arrow 1 refers to the reflected wave in the first interface, according to the simulated physical model (Fig. 4a), numbering 2 indicates the reflected wave in the second interface, and arrows 3 and 4 refer to the internal multiple.
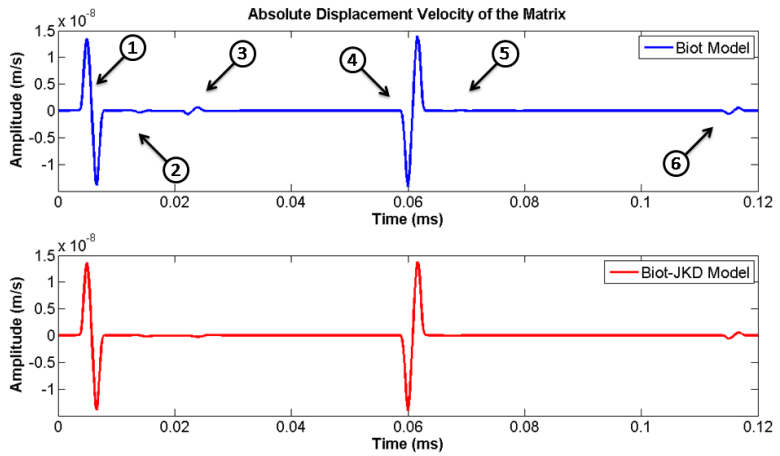

(a)
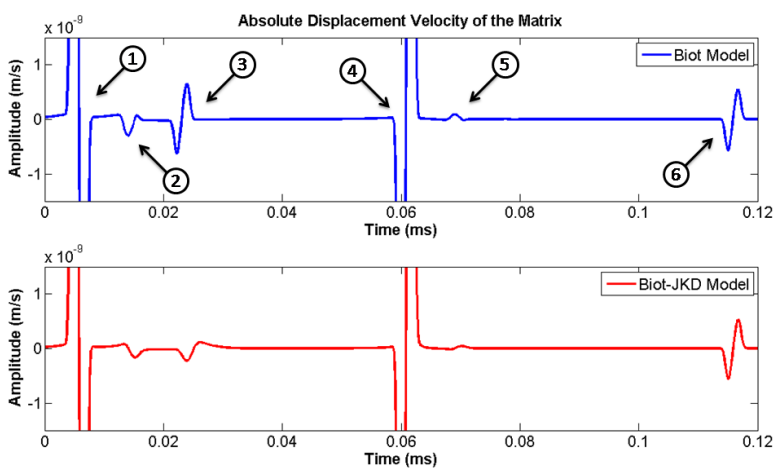

(b)

Figure 6 - Laboratory scale results (a) and the same with zoom in the vertical axis (b). Numbering 1 refers to the fast wave reflected on the first interface, according to the simulated physical model (Fig. 4b), arrow number 2 indicates the fast-slow or slow-fast converted wave, whereas the third arrow indicates the slow wave reflected in the first interface, the fast wave reflected in the second interface is indicated by the arrow number 4 , the fifth number indicates the slow wave that began its propagation from the source, but was converted to fast wave by refracting at the first interface and then reflected in the second interface and return to the surface, and the numbering 6 refers to the internal multiple of the fast wave.

main wave events in both low frequency and high frequency regimes in the reservoir and laboratory scales.

Some suggestions for future works:

- Analysis of the numerical stability of the algorithm in the case of 3-D stratified porous media.

- Evaluation of the results of physical modeling of the poroelastic waves propagation using the Biot and Biot-JKD equations.

\section{ACKNOWLEDGMENTS}

The authors are thankful to an anonymous reviewer for useful suggestions and comments. The authors also thank the Program for the Formation of Human Resources in Geophysics - 
PRH-PB 226 (UENF/PETROBRAS) for the financial support and the Laboratory of Engineering and Exploration of Petroleum (LENEP/CCT/UENF) for having provided the conditions for this work. The fourth author is a member of the National Institute of Science and Technology of Petroleum Geophysics (INCT-GP/CNPq/MEC), Brazil.

\section{REFERENCES}

AKKURATOV GV \& DMITRIEV VI. 1984. Method of calculation of a field of steady elastic vibrations in a layered medium. Comput. Math. Math. Phys., 24(1): 166-176. [In Russian].

ALLARD JF, DEPOLLIER C \& REBILLARD P. 1989. Inhomogeneous Biot waves in layered media. J. Acoust. Soc. Am., 66(6): 2278-2284.

AURIAULT J, BORNE L \& CHAMBON R. 1985. Dynamics of porous saturated media. J. Acoust. Soc. Am., 77: 1641-1650.

AZEREDO MM. 2013. Modelagem matemática e computacional da propagação de ondas sísmicas em meios poroelásticos estratificados. Ph.D. thesis. Universidade Estadual do Norte Fluminense Darcy Ribeiro, Campos dos Goytacazes, Brazil. 178 pp.

AZEREDO MM \& PRIIMENKO V. 2015. An algorithm for wave propagation analysis in stratified poroelastic media. Eurasian Journal of Mathematical and Computer Applications, 3(3): 4-15.

BAIRD GE, THOMAS PD \& SANG G. 1996. The propagation of elastic waves through a layered poroelastic medium. J. Acoust. Soc. Am., 99(6): 3385-3392.

BIOT MA. 1956. Theory of propagation of elastic waves in a fluid-saturated porous media. I. Higher frequency range. J. Acoust. Soc. Am., 28: 179-191.

BLANC E. 2013. Time-domain numerical modeling of poroelastic waves: the Biot-JKD model with fractional derivatives. Ph.D. thesis. AIX-Marseille Université, France. 158 pp.

BOUZIDI Y. 2013. Numerical models of converted slow P-wave modes in porous media. In: Fifth Biot Conference on Poromechanics. Vienna, Austria: ASCE.

BREKHOVSKIH LM. 1960. Waves in layered media. New York: Academic Press. $561 \mathrm{pp}$.

CARCIONE JM. 1996. Wave propagation in anisotropic, saturated porous media: plane-wave theory and numerical simulation. J. Acoust. Soc. Am., 99: 2655-2666.

CARCIONE JM. 2007. Wave fields in real media: wave propagation in anisotropic, anelastic and porous media. Handbook of Geophysical Exploration. Elsevier. 515 pp.
CARCIONE JM, MORENCY C \& SANTOS JE. 2010. Computational poroelasticity - a review. Geophysics, 75(5): 75A229-75A243.

FATIANOV AG. 1990. Semi-analytical method for the solution of direct dynamical problems in stratified media. Dokl. AN SSSR, p. 323-327. [In Russian].

FATIANOV AG \& MIKHAILENKO BG. 1988. A method for calculation of non-stationary wave fields in non-elastic stratified-inhomogeneous media. Dokl. AN SSSR, 301(4): 834-839. [In Russian].

FRENKEL J. 1944. On the theory of seismic and seismoelectric phenomena in a moist soil. J. Phys. (USSR), 3: 230-240.

HANYGA A \& LU JF. 2005. Wave field simulation for heterogeneous transversely isotropic porous media with the JKD dynamic permeability. Computational Mechanics, 36(3): 196-208.

HASKELL NA. 1953. The dispersion of surface waves on multilayered media. Bull. Seismol. Soc. Am., 43(1): 17-43.

JOHNSON DL, KOPLIK J \& DASHEN R. 1987. Theory of dynamic permeability and tortuosity in fluid-saturated porous media. Journal of Fluid Mechanics, 176(1): 379-402.

KARCHEVSKY AL. 2005a. Direct dynamic problem of seismicity for horizontally stratified media. Sib. Elect. Math. Reports, 2: 23-61. [In Russian].

KARCHEVSKY AL. 2005b. A numerical solution to a system of elasticity equations for layered anisotropic media. Russian Geology and Geophysics, 46(3): 339-351.

KUNETZ G \& D'ERCEVILLE. 1962. Sur certaines propriétés d'une onde acoustique plane de compression dans un milieu stratifié. Ann. Geophys., 18: 351-359.

LI Z, KOURI DJ \& CHESNOKOV EM. 2015. Time-domain solution of poroelastic wave equation with dynamic permeability. In: SEG New Orleans Annual Meeting. p. 3589-3593.

LORENZI A \& PRIIMENKO V. 2014. Direct problems for poroelastic waves with fractional derivatives. SIAM J. Math. Anal, 46(3): 3589-3593.

MASSON YJ \& PRIDE SR. 2010. Finite-difference modeling of Biot's poroelastic equations across all frequencies. Geophysics, 75(5): N33-N41.

MILANI M, MONACHESI L, SABBIONE JI, RUBINO JG \& HOLLIGER K. 2016. A generalized effective anisotropic poroelastic model for periodically layered media accounting for both Biot's global and interlayer flows. Geophysical Prospecting, 64(4): 1135-1148.

MIRANDA M. 2016. Método matricial em modelagem poroelástica: modelo de Biot-JKD. Master's dissertation. Universidade Estadual do Norte Fluminense Darcy Ribeiro, Campos dos Goytacazes, Brazil. $105 \mathrm{pp}$. 
MOLOTKOV LA. 1984. The Matrix method in the theory of wave propagation in layered elastic and fluid media. Leningrad: Nauka. 201 pp. [In Russian].

MOLOTKOV LA. 2002. On the matrix method in the theory of wave propagation in layered porous Biot media. Journal of Mathematical Sciences, 111(5): 3737-3749.

NIKOLAEVSKIY V. 2005. Biot-Frenkel poromechanics in Russia (Review). J. Eng. Mech., 131: 888-897.

OLIVEIRA IB. 2018. Modelagem de propagação das ondas elásticas em meios porosos 1D: modelos de Biot vs. Biot-JKD. Master's dissertation. Universidade Estadual do Norte Fluminense Darcy Ribeiro, Campos dos Goytacazes, Brazil. 139 pp.
PLONA TJ. 1980. Observation of a second bulk compressional wave in a porous medium at ultrasonic frequencies. Applied Physics Letters, 36(4): 259-261.

PRIDE SR \& CARAMBOIS S. 2005. Electroseismic wave theory of Frenkel and more recent developments. J. Eng. Mech., 131: 898-907.

THOMSON WT. 1950. Transmission of elastic waves through a stratified solid medium. J. Appl. Phys., 21: 89-93.

URSIN B. 1983. Review of elastic and electromagnetic waves propagation in horizontally layered media. Geophysics, 48: 1063-1081.

WHITE BS \& ZHOU M. 2006. Electroseismic prospecting in layered media. SIAM J. Appl. Math., 67(1): 69-98. 